\title{
Analysis of the Spatial-Frequency Characteristics of the Photo-Assisted Method of a Quartz Rough Surface Nano-Polishing
}

\author{
Vasyl Kanevskii, ${ }^{1}$ Serhii Kolienov, ${ }^{2}$ Valerii Grygoruk, ${ }^{2}$ Oleksandr Stelmakh, ${ }^{3}$ \\ and Hao Zhang ${ }^{3}{ }^{3}$ \\ ${ }^{1}$ Chuiko Institute of Surface Chemistry, NAS of Ukraine, Kyiv 03164, Ukraine \\ ${ }^{2}$ Taras Shevchenko National University of Kyiv, Kyiv 01601, Ukraine \\ ${ }^{3}$ Beijing Institute of Technology, Beijing 100081, China
}

Correspondence should be addressed to Hao Zhang; hao_zhang@bit.edu.cn

Received 10 April 2021; Revised 4 July 2021; Accepted 21 July 2021; Published 29 July 2021

Academic Editor: Giancarlo C. Righini

Copyright (c) 2021 Vasyl Kanevskii et al. This is an open access article distributed under the Creative Commons Attribution License, which permits unrestricted use, distribution, and reproduction in any medium, provided the original work is properly cited.

\begin{abstract}
The relationship between the spatial-frequency parameters of a rough surface with a random profile, which has a Gaussian form of the correlation function, and the amplitude-frequency characteristics of the electric field created by this surface is determined. The numerical determination of the evanescent field optimal configuration formed near the quartz rough surface in the gaseous medium saturated with chlorine molecules when illuminated from the quartz side has been considered. The finite-element approach is used to solve the Helmholtz two-dimensional vector equation. It was found that at the initial stage of photochemical polishing different electrodynamic conditions are created for the etching process depending on the profile height standard deviation value. In particular, when the standard deviation is less than $1 \mathrm{~nm}$, all surface protrusions, for which the spatial spectrum harmonics of the profile are located in the region of the maximum slope of the spectral function, are most actively etched. This leads to a decrease in the effective width of the spatial spectrum of a rough quartz surface and an increase in its correlation length. Therefore, simultaneously with decreasing the height of the protrusions, the surface becomes flatter. The paper shows the different character of quartz surface nano-polishing process conditions depending on the initial standard deviation of the profile height.
\end{abstract}

\section{Introduction}

Optics progress in such areas as fiber optics, holography, and integrated optics and photonics leads to a constant increase in requirements for both the quality of materials and the accuracy of the optical elements manufacture. Until recently, the tolerances provided by existing processing methods were more than sufficient for the formation of high-quality images, according to the Rayleigh criterion. However, today new optical technologies require that the deviations of the surface shape from the standard were much smaller than the wavelength of radiation [1]. In this case, an important characteristic of many optical elements is the level of surface roughness. At present, chemical-mechanical polishing methods [2] and vacuum plasma-chemical [3] etching are used to reduce surface roughness. A continuous search for new and more efficient ways of processing optical surfaces is in progress, among which we can highlight the method of using the optimal near-field [4] that is used for nano-polishing, such as quartz. In the general case, this method consists in immersing the rough surface of quartz in a medium containing molecular chlorine and creating an evanescent field over this surface, by irradiating it directly with light of a certain power and wavelength. The evanescent field near the surface, formed by irradiation, will be inhomogeneous due to the presence of protrusions and troughs on the surface. The electric component of the electromagnetic field is localized near the protrusions and has increased field strength compared to flat regions of the surface. The photodissociation of molecular chlorine near the protrusions occurs in regions of high electric field strength [5] with the formation of atomic chlorine and its ions, which are 
polarized in an external field and are attracted to surface protrusions, where they interact with quartz. This leads to local etching of the quartz protrusions surface, which reduces its roughness. It was shown in $[6,7]$ that the configuration of the evanescent field near the quartz surface significantly depends on the direction of radiation propagation and the refractive index of the medium above the quartz surface. This affects the efficiency of the nano-polishing process accordingly.

The paper [7] reports on the new photochemical polishing method using an alternative and more efficient approach compared to $[4,5]$. This approach makes it possible to additionally increase the electric field strength above the quartz surface in the region of protrusions in comparison with $[4,5]$ by using the effect of total internal reflection when light falls on the surface from the quartz side. In this case, the maximum field values and at the same time its optimal configuration from the point of view of photochemical polishing are achieved exclusively at the critical angle of light incidence [7]. In the approach under consideration, a sodium hypochlorite solution is used as a source of chlorine molecules, which is unstable, and in an acidic medium forms a significant fraction of molecular chlorine. At the same time, it is obvious that the intensity of the photodissociation of chlorine molecules and, accordingly, the rate of sub-nanopolishing of the quartz surface depends on the degree of excess of the level of the evanescent field strength over the threshold value. As a result, photodissociation of chlorine molecules takes place, and, therefore, the contrast of the electric field above the protrusions and troughs of the surface will also be an important parameter, at which the field strength in the region of the troughs remains below the threshold value and, accordingly, etching in the troughs does not occur. Thus, one of the actual problems is the possibility of increasing the contrast of the electric field near the quartz surface while holding the optimal configuration of this field for efficient etching of exclusively protrusions surface. One of the variants for increasing the contrast of the field, proposed in this work, can be an increase in the field intensity due to a higher impedance at the interface, on which the electromagnetic wave is incident. That can be achieved by replacing an aqueous solution of sodium hypochlorite in [7] with gaseous chlorine, the refractive index of which will be significantly lower than that of the solution. Obviously, this approach cannot be considered without taking into account the shape of the sample surface, the parameters of its roughness and the wavelength of radiation illuminating the surface. Therefore, it is important to consider in more detail the relationship between the field contrast near a random quartz surface described by a Gaussian correlation function and the contrasts generated by the spectral components of this profile. Since a random surface profile can be described by a set of spatial harmonics, a logical step in this direction is to choose a quartz surface with a sinusoidal profile as the object of study. The set of contrasts of each of the harmonics obtained in this way makes it possible to describe the spectral dependence of the field contrast for a random quartz surface described by a Gaussian correlation function.
Thus, the objectives of this work are (1) determination of the conditions for the formation of the optimal electric field, at which the most effective local photochemical etching of the sinusoidal surface protrusions will occur, leading to a decrease in its roughness level; (2) calculation of the field contrast directly above the surface of the sinusoidal profile depending on its spatial frequency and the height of the protrusions; and (3) finding the regularities of the influence of the parameters of a random quartz surface described by a Gaussian correlation function on the contrast value of the field formed by these irregularities, depending on the spatial frequency of the surface under consideration.

\section{Specificity of the Calculation Model}

The numerical calculation of the distribution of the electric field strength near the quartz surface, illuminated by directional monochromatic linearly polarized radiation, was performed similar to $[6,7]$. It was the basis for calculating the contrast of the electric field near the surface of quartz. We used special software developed by us that implements the solution of the Helmholtz equation by the finite-element method [8]. Verification of this software was carried out in the studies [6]. Figure 1 shows a computational cell in which the "quartz-chlorine gas" interface has a sinusoidal profile, where $h$ is the height of the surface protrusions.

The right and left sides of the calculation cell are limited by vertical lines, along which periodic boundary conditions are realized. Bulk quartz in region 2 has a refractive index $n_{2}=1.5168$. Chlorine gas in region 3 has a refractive index $n_{3}=1$. Losses in regions 2 and 3 are neglected. Absolutely absorbing layers are located below and above the computational cell in regions 1 and $4[9,10]$. The light source radiates a plane wave, which is polarized in the plane of incidence and falls on the "quartz-chlorine gas" interface. Its direction in Figure 1 is represented by the Poynting vector $\mathbf{P}_{i n}$, which is directed at an angle $\theta_{\text {in }}$ relative to the $Y$-axis, and the wave falls on the indicated interface from the side of quartz. We will also assume that the contrast $K$ of the evanescent field between the protrusions and troughs along the "quartz-chlorine gas" interface is determined by using the relation

$$
K=\frac{\left|E_{1}-E_{2}\right|}{\left(E_{1}+E_{2}\right)}
$$

where $E_{1}$ and $E_{2}$ are the amplitudes of the evanescent field in the regions of protrusions and troughs.

\section{Analysis of the Results}

Numerical calculations of the electric field strength along the surface of quartz with a sinusoidal profile were carried out for the case when a plane electromagnetic wave falls on the "quartz-chlorine gas" interface (Figure 1), the length of which in vacuum is $\lambda=500 \mathrm{~nm}$. At the same time, first of all, the question of the optimal configuration of the electric field strength over the protrusions and troughs of a sinusoidal surface was investigated, at which the field maxima are formed only above the surface of protrusions. Note that in 


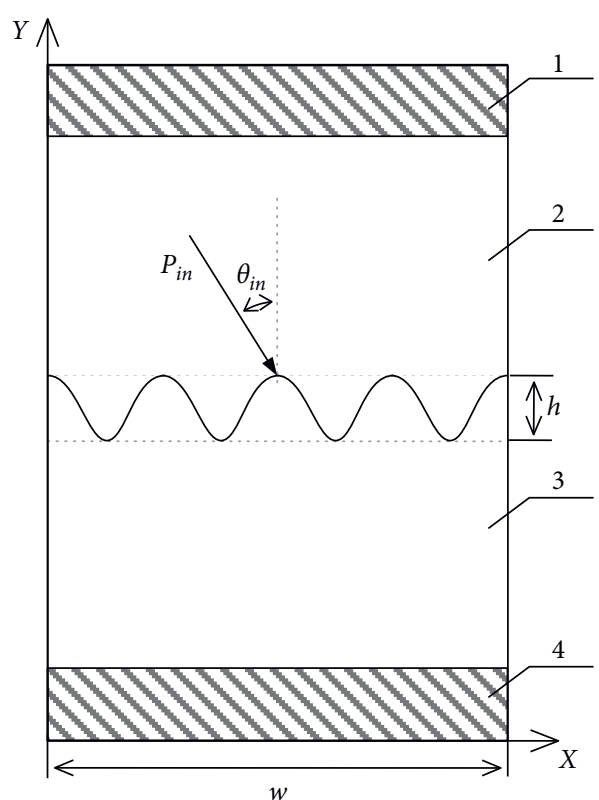

Figure 1: The 2D cell used to calculate evanescent field parameters in the near-field region of the nanoirregularities of the quartz surface. Regions 1, 2, 3, and 4 are, respectively, the regions of the upper absorbing layer, the quartz layer, the chlorine gas layer, and the lower absorbing layer.

[7] this problem has been investigated, but in the case when the profile of the quartz surface was a random function described by the Gaussian correlation function. It was found that the shape of the field above the random surface repeats the spatial profile of the quartz surface in the case when the quartz surface is illuminated from the side of the quartz at a critical angle $\theta_{c r}$. However, it was necessary to check this fact in the case when the profile of the quartz surface has a sinusoidal shape, and when its spatial frequency and the height of the protrusions are parameters.

Figures 2(a) and 2(b) show the distributions of the electric field modulus along the "quartz-chlorine gas" interface in the case when the quartz surface is illuminated at different angles $\theta_{i n}$ of the incident wave and in the case when the spatial frequency of the surface profile has the following values: $\quad \nu_{1}=0.8 \cdot 10^{-2} \mathrm{~nm}^{-1} \quad$ (Figure 2(a)) and $v_{2}=4 \cdot 10^{-2} \mathrm{~nm}^{-1}$ (Figure 2(b)).

At the same time, the heights of the profile protrusions are $4 \mathrm{~nm}$ (curves 1, 3, and 5 in Figure 2) and $16 \mathrm{~nm}$ (curves 2, 4 , and 6 in Figure 2). Curves 1 and 2, which are shown in Figures 2(a) and 2(b), correspond to the case when the illumination of a rough surface was carried out at a critical angle. Curves 3 and 4 , in turn, correspond to the case when $\theta_{i n}=30^{\circ}$. Similarly, curves 5 and 6 take place when $\theta_{\text {in }}=80^{\circ}$. Additionally, for comparison, Figure 2 shows a curve 7 , which represents a sinusoidal profile of the quartz surface with the protrusion height of $h=16 \mathrm{~nm}$. Analysis of curves 1 and 2, which are shown in Figures 2(a) and 2(b), as well as comparison of these curves with curve 7 , shows that the distribution of field maxima along the "quartz-chlorine gas" interface and the distribution of the tops of irregularities of the rough quartz surface coincide when illumination is carried out at a critical angle. In this case, an increase in the height of the irregularities of the quartz surface profile entails an increase in the field at the tops of these irregularities. When the shape of the field in the region of the tops begins to change more sharply (Figure 2(b)), this can lead to an inhomogeneous etching of the lateral surfaces of these irregularities. Analysis of curves 3 and 4 in Figures 2(a) and 2(b), in comparison with curve 7 , shows that we have a shift of the field maxima relative to the lateral surfaces of the surface profile protrusions at the angle of light incidence $\theta_{\text {in }}=30^{\circ}$. This means that in the photochemical polishing process at such angle of light incidence only a part of the lateral surface of the protrusions will be etched. That reduces the polishing efficiency. In this case, with an increase in the spatial frequency of the profile, the change in the field amplitude along the surface becomes more and more nonlinear with respect to the profile height. Comparison of curves 5 and 6 in Figures 2(a) and 2(b) with the surface profile curve 7 shows that the change in the electric field strength along the "quartz-chlorine gas" interface differs significantly from the surface profile shape. In this case, the field strength maxima appear in the areas of the surface troughs. It does not lead to surface polishing, but to an even greater increase in the depth of the ones and, accordingly, to an increase in the level of roughness. In addition, it is clearly seen that at the angle of light incidence $\theta_{\text {in }}=80^{\circ}$, the value of the field strength and the difference between the maxima and minima of the field significantly decrease in comparison with smaller angles of incidence. Thus, the results obtained allow us to state that only the critical angle of incidence is the most optimal for the photochemical polishing of quartz. Note also that, in the ideal case, the field in the troughs should not exceed the threshold value at which the photodissociation of chlorine molecules begins.

Let us first consider the contrast of the field over the sinusoidal profile depending on the height of the protrusions and the spatial frequency of the profile (provided $\theta_{i n}=\theta_{c r}$ ), before considering the dependencies of the electric field contrast over the random profile of the quartz surface on its spatial frequency. That will make it possible to analyze the frequency dependence of the field contrast $K(\nu)$ in the case when the profile of the quartz surface is random and described by a Gaussian correlation function.

Figure 3 shows the dependencies of the evanescent field contrast over the sinusoidal quartz surface profile depending on the spatial frequency of the profile at different heights of the protrusions: $5 \mathrm{~nm}$ (curve 1), $50 \mathrm{~nm}$ (curve 2), and $150 \mathrm{~nm}$ (curve 3). Note that the spatial frequency of the profile determines the width of the protrusions, which corresponds to the period $T$ of the sinusoidal profile. It is clearly seen from the presented curves that for low heights, $h=5 \mathrm{~nm}$ (curve 1), the contrast of the field $K_{\text {sin }}$ between the protrusions and troughs of the sinusoidal profile changes in direct proportion to the increase in the profile frequency. In this case, the increase in contrast in the range of spatial frequencies considered in Figure 3 is primarily due to an increase in the field strength at the tops of the protrusions, when their radius of curvature decreases with decreasing width $T$ of the protrusions, and $h \ll T[11]$. At the same time, 


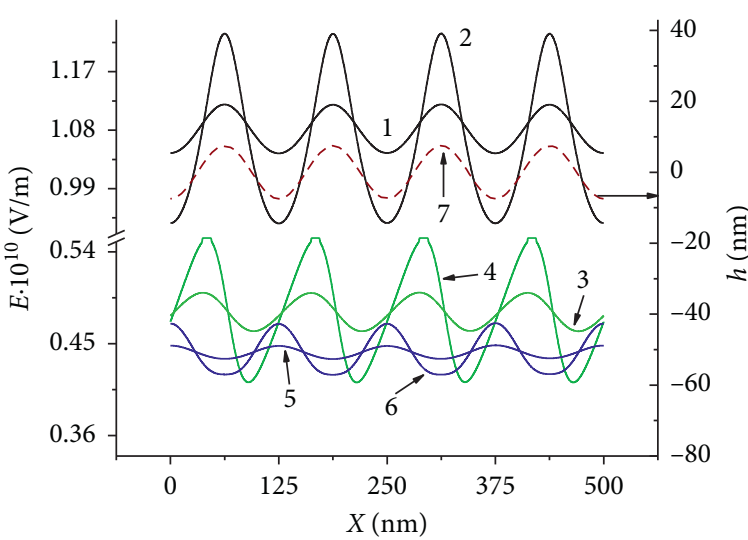

(a)

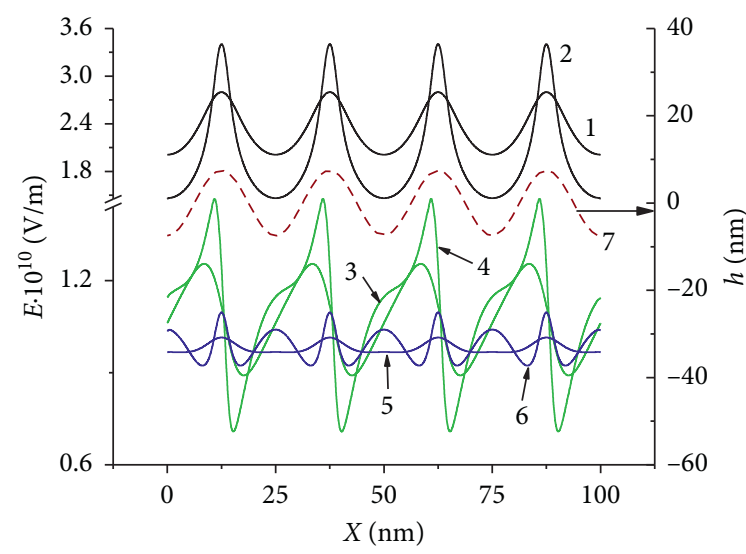

(b)

FIGURE 2: Distributions of the modulus of the electric field along the "quartz-chlorine gas" interface, which has a sinusoidal profile with spatial frequencies $\nu_{1}=0.8 \cdot 10^{-2} \mathrm{~nm}^{-1}$ (a) and $\nu_{2}=4 \cdot 10^{-2} \mathrm{~nm}^{-1}$ (b) at different angles of the incident wave: $\theta_{\text {in }}=42.5^{\circ}$ (curve 1 corresponds to $h=4 \mathrm{~nm}$ and curve 2 corresponds to $h=16 \mathrm{~nm}$ ); $\theta_{\text {in }}=30^{\circ}$ (curve 3 corresponds to $h=4 \mathrm{~nm}$ and curve 4 corresponds to $h=16 \mathrm{~nm}$ ), $\theta_{\text {in }}=80^{\circ}$ (curve 5 corresponds to $h=4 \mathrm{~nm}$ and curve 6 corresponds to $h=16 \mathrm{~nm}$ ). The wavelength of the incident radiation (in vacuum) is $\lambda=500 \mathrm{~nm}$. The protrusion height of the sinusoidal profile is $h=16 \mathrm{~nm}$ (curve 7).

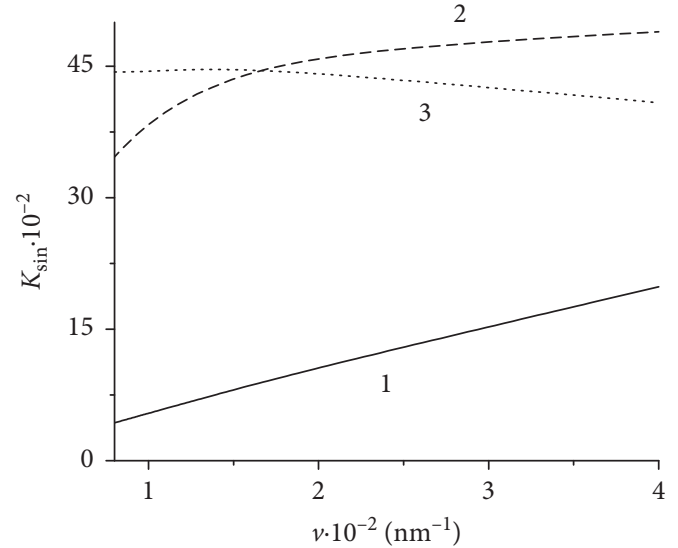

Figure 3: Contrast of the evanescent field $K_{\text {sin }}$ over the sinusoidal quartz surface profile depending on the spatial frequency $v$ of the profile at different heights of the protrusions $h: 5 \mathrm{~nm}$ (curve 1), $50 \mathrm{~nm}$ (curve 2), and $150 \mathrm{~nm}$ (curve 3).

the effect of changing the width of the protrusions in the considered range of spatial frequencies on the radius of curvature of the surface at the top of the protrusions changes with an increase in the height $h$ of the protrusions. As a consequence, as the spatial frequency increases, the growth in the field contrast slows down (curve 2, Figure 3 ) and tends to a constant value. However, with an even greater increase in the height of the protrusions, $h=150 \mathrm{~nm}$ (curve 3), a slow decrease in contrast is observed with an increase in the spatial frequency. In this case, the protrusions become similar to waveguides with a decreasing aperture towards the top. Decreasing the width of the protrusions with increasing spatial frequency leads to a decrease in the waveguide aperture and, as a consequence, a decrease in the field near the top of the ones [12], which in turn leads to a decrease in field contrast.

Let us now consider the dependencies of the evanescent field contrast over the sinusoidal profile of the quartz surface on the height of the protrusions of this profile (Figure 4) at different spatial frequencies the profile $v: 0.8 \cdot 10^{-2} \mathrm{~nm}^{-1}$ (curve 1), $1.6 \cdot 10^{-2} \mathrm{~nm}^{-1}$ (curve 2), and $4 \cdot 10^{-2} \mathrm{~nm}^{-1}$ (curve $3)$. In Figure 4, three regions can be distinguished: the region of small values of the parameter $h(h \leq 30 \mathrm{~nm})$, where the field contrast increases in proportion to the height of the protrusion; the region of large parameter values $h$ $(h \geq 70 \mathrm{~nm})$; and the transition region $(30 \leq h \leq 70 \mathrm{~nm})$. In this case, the behavior of the field contrast $K_{\text {sin }}$ in the regions corresponding to small and large changes in the parameter $h$ is explained by the same reasons that were described above in Figure 3. In fact, the regions of large and small values of the parameter $h$ determine two main characteristics of contrast, which affect the dynamics and nature of quartz etching in the process of changing the roughness parameters of the surface profile.

The dependencies shown in Figures 3 and 4 allow a more detailed analysis of the relationship between the field contrasts near a random quartz surface described by a Gaussian correlation function and the contrasts generated by the spatial spectral components of this profile. Consider the relationship between the spatial energy spectrum of a rough surface and the roughness parameters of this surface as a first step. If we assume that the profile of the quartz surface is described by a Gaussian correlation function with a given correlation length and standard deviation, then this profile can be represented by a set of sinusoidal surfaces with the protrusions height, which is determined as [13]

$$
h(\nu)=4|S(\nu)|,
$$

where $|S(\nu)|$ is the modulus of the function of the spatial spectrum of the surface, which is described by the Gaussian correlation function, and $v$ is the spatial frequency of the profile. Thus, it is possible to study the contribution of the individual components of the spatial spectrum of a random surface to the contrast of the field $K(\nu)$ above its protrusions 


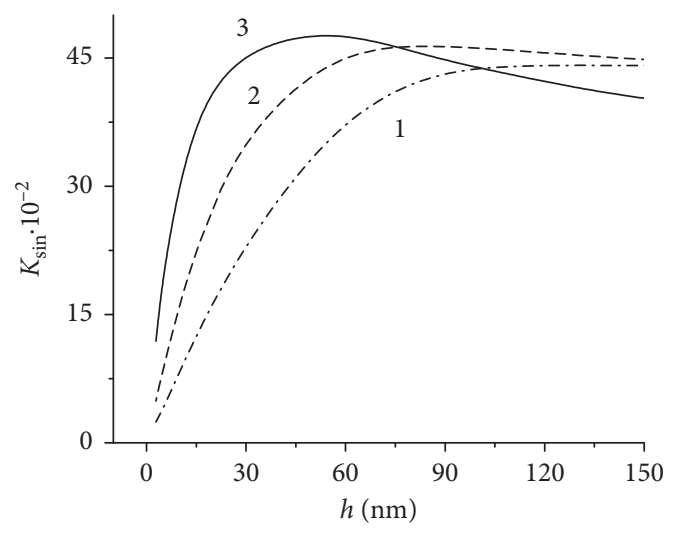

Figure 4: The contrast $K_{\text {sin }}$ of the evanescent field over the sinusoidal quartz surface profile depending on the height of the protrusions of this surface $h$ at different spatial frequencies of the profile $v$ : $0.8 \cdot 10^{-2} \mathrm{~nm}^{-1}$ (curve 1), $1.6 \cdot 10^{-2} \mathrm{~nm}^{-1}$ (curve 2), and $4 \cdot 10^{-2} \mathrm{~nm}^{-1}$ (curve 3 ).

and troughs by calculating the dependence for each sinusoidal profile $K_{\sin }(\nu)$ with the frequency $v$ and height $\delta=1$ of the protrusions. Figure 5 shows the modulus of the functions of the spatial spectrum of the surface, which are described by a Gaussian correlation function, when standard deviation is $\delta=1 \mathrm{~nm}$ and correlation length are $\sigma=14.2 \mathrm{~nm}$ (curve 1), $\sigma=23.7 \mathrm{~nm}$ (curve 2), and $\sigma=33.1 \mathrm{~nm}$ (curve 3). Thus, the field contrast curves $K(\nu)$ were obtained along the random quartz surfaces for each of the functions shown in Figure 5 (but with its own parameter $\delta$ ) and are shown in Figure 6.

Figure 6 shows the contrast $K$ distributions of the evanescent field over irregularities of a random surface profile with a Gaussian correlation function, depending on the spatial frequency $v$ of this profile for cases where the profile correlation lengths correspond to $\sigma=14.2 \mathrm{~nm}$ (curves 1, 4, and 7), $\sigma=23.7 \mathrm{~nm}$ (curves 2, 5, and 8), and $\sigma=33.1 \mathrm{~nm}$ (curves 3, 6, and 9), and the standard deviation of the profile height corresponds to $\delta=8 \mathrm{~nm}$ (curves 1,2 , and 3 ), $\delta=4 \mathrm{~nm}$ (curves 4, 5, and 6), and $\delta=1 \mathrm{~nm}$ (curves 7, 8, and 9 ). The dependencies $K(\nu)$ were obtained from numerical calculations of the distribution of the electric field strength along the quartz surface for the case when a plane electromagnetic wave with a length of $\theta_{c r} \approx 42.5^{\circ} \mathrm{nm}$ in vacuum falls on the interface at the critical angle $\theta_{c r} \approx 42.5^{\circ}$ of total internal reflection.

Curves 1-3 in Figure 6 describe the contrast of the field formed by three random profiles, with different correlation lengths, the root-mean-square deviation of which is $\delta=8 \mathrm{~nm}$. A feature of these curves is the fact that their spectral distribution of the field contrast $K(\nu)$ is practically uniform in the frequency range where the main energy of the spatial spectrum of the surface is concentrated (within its effective width) (Figure 5). This means that the field contrast within the given frequencies does not depend on the amplitude of the spatial spectrum harmonic, that is, from the height of the protrusions of the sinusoidal profile. For example, as seen in Figure 5, at a correlation length of $\sigma=14.2 \mathrm{~nm}$, the width of the spatial spectrum function $|S(\nu)|$

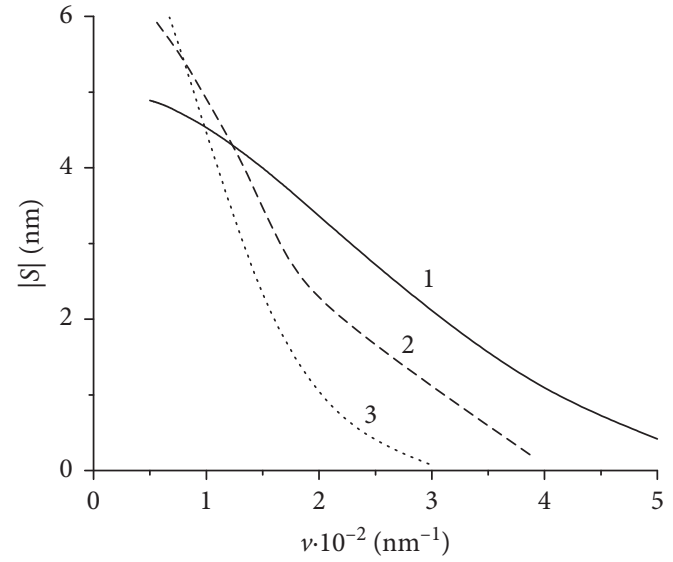

FIgURE 5: The module of the spatial spectrum functions for the random surface profiles characterized by a Gaussian correlation function with a correlation length of $\sigma=14.2 \mathrm{~nm}$ (curve 1), $\sigma=23.7 \mathrm{~nm}$ (curve 2), and $\sigma=33.1 \mathrm{~nm}$ (curve 3).

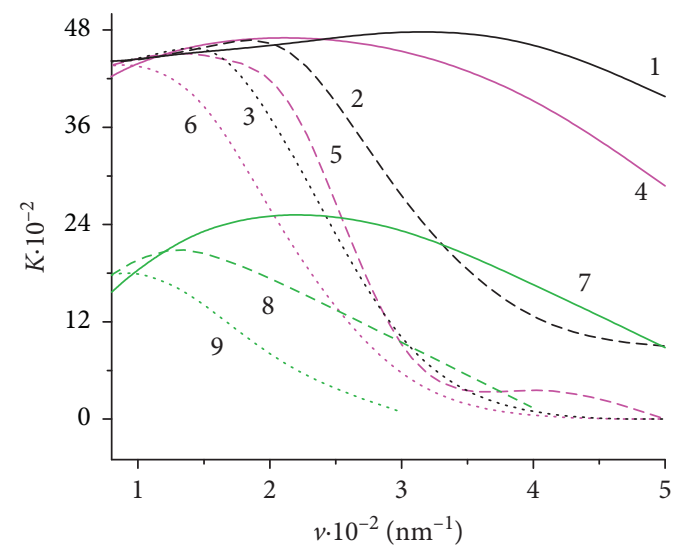

Figure 6: Contrast $K$ of the evanescent field over irregularities of a random surface profile with a Gaussian correlation function, depending on the spatial frequency $v$ of this profile for cases when the profile correlation lengths correspond to $\sigma=14.2 \mathrm{~nm}$ (curves 1 , 4, and 7), $\sigma=23.7 \mathrm{~nm}$ (curves 2, 5, and 8), and $\sigma=33.1 \mathrm{~nm}$ (curves 3 , 6, and 9), and the standard deviation of the profile height corresponds to $\delta=8 \mathrm{~nm}$ (curves 1,2, and 3), $\delta=4 \mathrm{~nm}$ (curves 4, 5, and 6 ), and $\delta=1 \mathrm{~nm}$ (curves 7,8 , and 9).

at the $1 / e$ level from the maximum is determined by the harmonic with a frequency $v_{m} \approx 3.5 \cdot 10^{-2} \mathrm{~nm}^{-1}$, for which the height of the sinusoidal profile protrusions exceeds $50 \mathrm{~nm}$. Moreover, for curve 1 in Figure 6 in the frequency range up to frequency $\delta=8$, the contrast changes slightly, which is in a good agreement with the data in Figure 4, where for all sinusoids with a protrusion height more than $50 \mathrm{~nm}$ and spatial frequencies less than $\delta=8$, the contrast with height also practically does not change. Similar results are observed for curves 2 and 3 in Figure 6. Thus, with a sufficiently large value of the standard deviation of the height of the random profile $(\delta=8 \mathrm{~nm})$, the quartz surface will be etched in the region of all protrusions almost uniformly, which will lead to a decrease in the standard deviation while maintaining the current correlation length of the profile. However, as curves 4-6 show in Figure 6, when the height of 
the protrusions decreases to a level at which the standard deviation of the profile height is $\delta=4 \mathrm{~nm}$, the distribution of the contrast level within the effective width of the spatial spectrum of the profile becomes less uniform. This unevenness increases with a decrease in the height of the protrusions, which is well shown by curves 7-9 in Figure 6 obtained for the case when the standard deviation of the profile height is $\delta=1 \mathrm{~nm}$. In this case, a maximum appears on the spectral contrast curves and its position corresponds to the frequencies where the spatial spectrum function (Figure 5) has the maximum slope.

This means that with a decrease in the height of the protrusions in the photochemical etching process, the contrast of the field near the quartz surface is redistributed in such a way that further etching leads not only to a decrease in the standard deviation of the profile height, but also to a decrease in the spectrum width, and hence to an increase in the profile correlation length.

Moreover, as follows from Figure 4 (the region where $h \leq 50 \mathrm{~nm}$ ), as well as curves 7-9 in Figure 6 (the region where $v \leq 2 \cdot 10^{-2} \mathrm{~nm}^{-1}$ ), the field contrast begins to depend on both the surface profile height and the correlation length, which in both cases leads to a decrease in contrast and, as a consequence, to a decrease in the etching intensity. As a result, the peaks of curves 7-9 shown in Figure 6, decrease and shift to the left. In this case, it is obvious that in the process of further etching, the surface will become flatter due to an increase in the correlation length and its level of roughness will decrease.

\section{Conclusions}

It has been established that the best conditions for photochemical polishing of a quartz surface are created only at a critical angle of light incidence on the "quartz-chlorine gas" interface. In this case, the maxima of the electromagnetic field strength are formed exclusively above the surface protrusions, and the field minima are formed in the troughs, which makes the surface polishing process as efficient as possible, since only the surface protrusions are etched.

The dependence of the field contrast above the sinusoidal profile of the surface on the spatial frequency of the given profile at low values of the profile in homogeneities height ( $h \leq 5 \mathrm{~nm}$ ) has a linearly increasing character; at its large values $(h \geq 50 \mathrm{~nm})$, the field contrast practically does not change.

The dependencies of the field contrast on the height of the inhomogeneities of the sinusoidal profile in the considered range of spatial frequencies $(0.8-4) \cdot 10^{-2} \mathrm{~nm}^{-1}$ are divided into two regions differing in a fundamentally different nature of etching of surface irregularities: linearly increasing region $(h \leq 30 \mathrm{~nm})$ and region with practically constant contrast values $(h \geq 70 \mathrm{~nm})$.

The initial stage of the quartz surface nano-polishing process has a different character depending on the value of the initial standard deviation of the profile height. At sufficiently high levels of surface roughness, when the height of the protrusions exceeds about $50 \mathrm{~nm}$, at the initial stage the etching of the quartz surface occurs uniformly, regardless of the spatial frequency and height of the protrusions. In the process of such nano-polishing, the height of the protrusions will decrease while maintaining the shape of the spatial spectrum function. That is, the width of the spectrum and, consequently, the correlation length of the profile of the quartz surface will not change. In the case of low protrusion heights (less than $50 \mathrm{~nm}$ ), nano-polishing leads to an increase in etching unevenness. Those harmonics of the spatial spectrum of the surface where the spectral function has the maximum slope will begin to etch most intensively. This will lead to a decrease in the effective spectrum width and, as a consequence, to an increase in the correlation length of a rough surface. Thus, the quartz surface will become flatter with a simultaneous decrease in the height of the surface protrusions; that is, its level of roughness will decrease.

\section{Data Availability}

The data used to support the findings of this study are available from the corresponding author upon request.

\section{Conflicts of Interest}

None of the authors have any conflicts of interest regarding the publication of this paper.

\section{References}

[1] G. Ghosh, A. Sidpara, and P. P. Bandyopadhyay, "Review of several precision finishing processes for optics manufacturing," Journal of Micromanufacturing, vol. 1, no. 2, pp. 170-188, 2018.

[2] L. Cook, Chemical Mechanical Planarization (CMP), Digital Encyclopedia of Applied Physics, Wiley Online Library, Hoboken, NJ, USA, pp. 1-38, 2019.

[3] F. Machalett and P. Seidel, Focused Ion Beams and Some Selected Applications, Digital Encyclopedia of Applied Physics, Wiley Online Library, Hoboken, NJ, USA, pp. 1-39, 2019.

[4] W. Nomura, T. Yatsui, and M. Ohtsu, "Nonadiabatic nearfield optical polishing and energy transfers in spherical quantum dots," Springer Series in Optical Sciences, vol. 155, pp. 113-130, 2010.

[5] M. Naruse, Ohtsu M 2014 Dressed Photons, Springer, Berlin, Germany, 2014.

[6] V. I. Kanevskii and S. O. Kolienov, "Analysis of electrodynamic conditions of photo-assisted nanoscale polishing of silica covered with calcium hypochlorite: theoretical analysis," Journal of Modern Optics, vol. 67, no. 3, pp. 242-251, 2020.

[7] V. I. Kanevskii and S. O. Kolienov, "Technique of lightassisted polishing of quartz surface covered with sodium hypochlorite solution: electrodynamical analysis," Journal of Modern Optics, vol. 67, no. 7, pp. 647-653, 2020.

[8] J. Jin, The Finite Element Method in Electromagnetics, John Wiley \& Sons, New York, NY, USA, 2002.

[9] W. C. Chew and W. H. Weedon, "A 3D perfectly matched medium from modified maxwell's equations with stretched coordinates," Microwave and Optical Technology Letters, vol. 7, no. 13, pp. 599-604, 1994.

[10] Z. S. Sacks, D. M. Kingsland, R. Lee, and J.-F. Lee, "A perfectly matched anisotropic absorber for use as an absorbing boundary condition," IEEE Transactions on Antennas and Propagation, vol. 43, no. 12, pp. 1460-1463, 1995. 
[11] Y. Fu, P. Zhang, J. P. Verboncoeur, A. J. Christlieb, and $\mathrm{X}$. Wang, "Effect of surface protrusion on plasma sheath properties in atmospheric microdischarges," Physics of Plasmas, vol. 25, no. 1, Article ID 013530, 2018.

[12] A. J. L. Adam, "Review of near-field terahertz measurement methods and their applications," Journal of Infrared, Millimeter, and Terahertz Waves, vol. 32, no. 8-9, pp. 976-1019, 2011.

[13] H. Raether, Surface Plasmons on Smooth and Rough Surfaces and on Gratings, Springer, Berlin, Germany, 1988. 